\title{
A Link between c-Myc-mediated Transcriptional Repression and Neoplastic Transformation
}

\author{
Linda A. Lee, ${ }^{\star}$ Christine Dolde, ${ }^{\S}$ John Barrett, ${ }^{\star}$ Chyi S. Wu, ${ }^{\star}$ and Chi V. Dang ${ }^{\star \neq \S}$ \\ $*$ Departments of Medicine, ${ }^{\ddagger}$ Molecular Biology \& Genetics, ${ }^{\S}$ Program in Human Genetics \& Molecular Biology, and $\|^{\text {The Johns Hopkins }}$ \\ Oncology Center, The Johns Hopkins University School of Medicine, Baltimore, Maryland 21205
}

\begin{abstract}
Recent studies indicate that the transcription factor c-Myc contributes to oncogenesis by altering the expression of genes involved in cell proliferation, but its precise function in neoplasia remains ambiguous. The ability of c-Myc to bind the sequence $\mathrm{CAC}(\mathrm{G} / \mathrm{A}) \mathrm{TG}$ and transactivate appears to be linked to its transforming activity; however, c-Myc also represses transcription in vitro through a pyrimidinerich cis element termed the initiator (Inr). In transfection experiments using the adenoviral major late (adML) promoter, which contains two Myc binding sites and an Inr, we determined that c-Myc represses transcription through the initiator in vivo. This activity requires the dimerization domain and amino acids 106 to 143, which are located within the transactivation domain and are necessary for neoplastic transformation. We studied a lymphoma-derived c-Myc substitution mutation at 115 -Phe, which is within the region required for transcriptional suppression, and found the mutant more effective than wild-type c-Myc in transforming rodent fibroblasts and in suppressing the adML promoter. Our studies of both loss-of-function and gain-of-function c-Myc mutations suggest a link between c-Myc-mediated neoplastic transformation and transcriptional repression through the Inr. (J. Clin. Invest. 1996. 97:1687-1695.) Key words: transrepression - bHLH • basic-helix-loop-helix • Burkitt lymphoma
\end{abstract}

\section{Introduction}

Deregulated c-myc gene expression is a frequent finding in many animal and human neoplasms (1). In certain cancers, such as human Burkitt lymphomas, genetic alterations of c-myc that result in its deregulated expression are sine qua non. Recognition of c-Myc protein function is a prerequisite for understanding its role in oncogenesis. A better understanding of c-Myc function has recently emerged from studies indicating that c-Myc is a member of the basic-helix-loop-helix leucine zipper (bHLHLZ) family of transcription factors. Mutational analysis uncovered several functional domains in-

Address correspondence to Dr. Chi V. Dang, Ross Research Building, Room 1025, The Johns Hopkins University School of Medicine, 720 Rutland Avenue, Baltimore, MD 21205. Phone: 410-955-2773; FAX: 410-955-0185.

Received for publication 11 September 1995 and accepted in revised form 19 January 1996.

J. Clin. Invest.

(C) The American Society for Clinical Investigation, Inc.

0021-9738/96/04/1687/09 \$2.00

Volume 97, Number 7, April 1996, 1687-1695 cluding an $\mathrm{NH}_{2}$-terminal transactivation domain and a COOH-terminal dimerization domain, which were both shown to be necessary for c-Myc-mediated transformation (2-7). c-Myc dimerizes with another bHLHLZ protein, Max, and the heterodimer recognizes a core nucleotide sequence, $\mathrm{CAC}(\mathrm{G} / \mathrm{A}) \mathrm{TG}$, also referred to as the enhancer box $(\text { E-box })^{1}(8,9)$. c-Mycmediated transactivation of artificial reporter constructs driven by multiple copies of the E-box has been observed by many investigators (9-14).

Several issues contribute to the complexity of c-Myc-mediated transactivation, however. First, Max not only dimerizes with Myc to form activating dimers, but Max also forms homodimers $(15,16)$ and dimers with two recently identified proteins, Mad (17) and Mxi-1 (18). Unlike Myc-Max heterodimers, Max-Max, Max-Mad, and Max-Mxi1 dimers have been shown to inhibit E-box-driven transactivation or cell transformation by c-Myc $(11,17,19,20)$. The fact that Max is expressed constitutively whereas Myc and Mad expression are cell growth dependent $(4,21-23)$ suggests that changing ratios of activating dimers to inhibitory dimers with respect to cell proliferation may be a form of transcriptional regulation by c-Myc. A second issue is that c-Myc may interact with transcriptional regulatory proteins other than Max. The $\mathrm{NH}_{2}$-terminal domain has been reported to interact directly with TATA-binding protein $(24,25)$ or the retinoblastoma-related protein p107 (26), whereas the $\mathrm{COOH}$ terminus may bind to Yin-Yang-1 (YY1) (27) or TFII-I (28). The biological relevance of these interactions in vivo remains to be elucidated. Third, there is significant variability in the degree of E-boxdriven transactivation reported in the literature $(11,29,30)$. The inverse correlation between endogenous c-Myc levels with cell proliferation may explain some of the observed variation in the degrees of c-Myc-mediated transactivation in transfection experiments. Variations in endogenous levels of c-Myc that occur with changes in cell density, a parameter not rigorously controlled in most transfection experiments, appear to affect E-box-driven transactivation by c-Myc because the rate of cell proliferation varies inversely with the degree of cell density $(31,32)$. Finally, the observation that a nontransforming c-Myc mutant with a deletion of amino acids 106-143 of the transactivation domain retains the ability to transactivate conflicts with the hypothesis that c-Myc-mediated transactivation is linked to transformation (14). This observation implies a dissociation between the transactivating and transforming properties of c-Myc and raises the possibility of the existence of other mechanisms of transformation by c-Myc.

Although the transactivating properties of c-Myc have been best described, c-Myc-mediated transcriptional repres-

1. Abbreviations used in this paper: adML promoter, adenoviral major late promoter; E-box, enhancer-box; HLH, helix-loop-helix; Inr, initiator element; MLV, Moloney murine leukemia virus. 
sion has also been reported $(29,30,33,34)$. In the course of studying c-Myc-mediated transactivation in our laboratory, an myc expression plasmid repressed an adenoviral major late (adML) promoter driven reporter in cells at low density. Unlike artificial constructs (composed simply of multimeric repeats of the E-box driving a reporter gene) frequently used to study c-Myc-mediated transactivation, the adML promoter contains an initiator element (Inr) that follows the consensus, YAYTCYYY (Y = pyrimidine base). The initiator element has been identified in several TATA-less promoters of genes that are regulated during differentiation $(35,36)$. In vitro transcription experiments show that Myc represses initiator driven transcription by TFII-I, a ubiquitous transcription factor that binds the Inr in gel shift assays (28). We now describe experiments demonstrating that $\mathrm{c}-\mathrm{Myc}$ represses transcription through the Inr in vivo. During the course of our work, several other studies that reached similar conclusions on c-Myc-mediated transcriptional suppression were reported (37-39). In the current study, we also determined which c-Myc functional domains participate in c-Myc-mediated repression through the Inr and identified a critical region, amino acids 106-143, within the transactivation domain that is required for repression through the Inr and neoplastic transformation. We observed that a lymphoma-derived mutant Myc protein, which contains a substitution of 115-Phe within the region required for repression, repressed the adML promoter more dramatically and conferred a greater cell growth potential than wild-type c-Myc, suggesting that repression may be another important mechanism by which c-Myc transforms cells.

\section{Methods}

Construction of plasmids. pMLPCAT and pMLPLuc are plasmids composed of the adML promoter driving the chloramphenicol acetyltransferase or luciferase genes, respectively. To generate pMLPCAT, the adenoviral major late promoter was isolated from the plasmid, pHTXB (gift of J. Corden, Johns Hopkins University, Baltimore, $\mathrm{MD}$ ) and subcloned into the XbaI/SmaI sites of pE1bCAT. The same fragment was cloned into pGL2Basic (a luciferase reporter plasmid; Promega Corp., Madison, WI) to obtain the pMLPLuc construct.

The adML promoter was mutated in the cloning vector pBSIIKS $^{-}$. To mutate the initiator element, a pair of 28-bp oligonucleotides spanning the initiator element and containing the desired mutation (top strand sequence: 5'-TCCTCACATACTTCCGCATCGCTGTCTG-3') were generated and used in the PCR with two flanking primers corresponding to pBSIIKS $^{-}$vector sequences. The mutated MLP fragment was subcloned into the XhoI/SmaI sites of pE1bCAT and sequenced for verification. The same fragment was cloned into $\mathrm{pGL} 2 \mathrm{~B}$ asic to create the $\mathrm{p} \Delta \mathrm{InrLuc}$ plasmid.

Similarly, the two E-boxes were mutated to generate the reporter construct DMCAT, in which the initiator element is intact. The M2 site was mutated using a pair of primers overlapping the M2 site creating an SalI site within M2: 5'-TAGGCGTCGACACCGGGTGTTCCTGAA; 3'-CCAAATATCCACATCCGCAGCTGTGGC. The mutated PCR fragment was then used in a PCR reaction with a second set of primers to create an XhoI site within M1: 5'-AAGACTCGAGTCGCCCTCTTCGGCATC; 3'-GAGGTCCCACACTTCTGAGCTCAGCGG. The double mutant fragment was subcloned into the XhoI/SmaI sites of pE1bCAT and sequenced for verification.

pMyc designates the plasmid composed of the full-length c-myc cDNA driven by the Rous-Sarcoma LTR. p $\Delta 41-53$, p $\Delta 55-92$, p $\Delta 93-$ 103, p $\Delta 106-143$, and $\mathrm{p} \Delta \mathrm{HLH}$ (gifts of W. Lee, University of Pennsylvania, Philadelphia, PA) are comprised of the Moloney murine leukemia virus (MLV) LTR driving a 2.7-kb genomic myc fragment or $m y c$ mutants as described (5). For the experiments designed to assess the transcriptional activity of the mutant $\mathrm{MycB} 2$, a genomic myc expression plasmid driven by the MLV LTR was used both for the transfection experiments and for subcloning the PstI-PstI fragment from pAMPB2, a gift of T. Yano and M. Raffeld (National Cancer Institute, Bethesda, MD [40]).

Cell cultures and transfection. Mouse $\mathrm{L}$ fibroblasts were seeded to $20 \%$ confluency $\left(2.9 \times 10^{6}\right.$ cells per $100-\mathrm{mm}$ dish $)$ and transfected using the DEAE dextran method (41) pBluescript or pMLV (an empty expression plasmid) was added to control for the total amount of DNA added to each plate. $16 \mathrm{~h}$ later, a $10 \% \mathrm{DMSO} /$ chloroquine shock was performed (41). CAT activity was assayed by the simple phase extraction method (42). Luciferase activity was measured using the luciferase assay system (Promega Corp.). Data was normalized for total protein as measured by the method of Bradford (43).

Rat 1a fibroblasts were transfected with pMLV, pMycB2, or pMyc, and a hygromycin resistance marker plasmid using Lipofectin (GIBCO BRL, Gaithersburg, MD) as described (44). Transformed cells were selected with $80 \mu \mathrm{g} / \mathrm{ml}$ hygromycin B (Sigma Chemical Co., St. Louis, MO), and over 100 hygromycin resistant colonies were pooled. Total cell lysates were prepared in $2 \times$ Laemmli buffer (45). Proteins from the cell lysates were resolved by SDS/10\% PAGE and subjected to immunoblot analysis using a monoclonal mouse antibody $9 \mathrm{E} 10$ (46). The blot was incubated with secondary goat anti-mouse horseradish peroxidase-conjugated antibody (Bio-Rad Laboratories, Rockville Centre, NY), and reactive polypeptides were detected by the enhanced chemoluminescence system (Amersham Corp., Arlington Heights, IL). The soft agar anchorage-independent growth assay was performed as previously described (44). $1.2 \times 10^{5}$ single cells in suspension were plated in $0.7 \%$ agarose on $100-\mathrm{mm}$ dishes, incubated at $37^{\circ} \mathrm{C}$, and dishes were photographed at $16 \mathrm{~d}$.

Rat embryo cell cotransformation assays using the activated pEJras plasmid were performed as described in our previous publication (3). Transformed foci were counted $2 \mathrm{wk}$ after lipofection.

\section{Results}

c-Myc represses transcription from the adML promoter through the Inr element. In our earlier study, it was observed that at low cell density exogenous c-Myc did not affect basal E-box-driven transcription from an artificial reporter construct lacking an Inr presumably because endogenous Max levels are limiting (31). However, exogenous c-Myc at low cell density repressed an adML promoter driven reporter gene, suggesting that the repressive effect may be exerted by Myc through a promoter element other than an E-box. The adML promoter contains an Inr located 23 bp downstream from the TATA box (Fig. 1). Two E-boxes also exist upstream. Based on the report that Myc could bind to the Inr and inhibit TFII-Idriven transcription (28), we tested if c-Myc could repress through the Inr in vivo. Mouse L cells grown to $20 \%$ cell confluency were cotransfected with increasing concentrations of pMyc, a Myc expression plasmid, and pMLPCAT, a reporter plasmid composed of the adML promoter driving the chloramphenicol acetyltransferase gene. Titration with increasing amounts of $\mathrm{pMyc}$ resulted in an initial transactivation of pMLPCAT followed by a decline to basal levels at the highest input pMyc (Fig. 1). Trends in transcriptional activity were emphasized because our earlier experiments indicated that variability in transcriptional activity is related to the ability of exogenous Myc to titrate endogenous levels of Max (31). Despite rigorous technical effort, variability in reporter activity between different transfection experiments was observed. This variability, also noted by others (30), led us to report here the average fold change in transcriptional activity from several experiments rather than representative data. The 

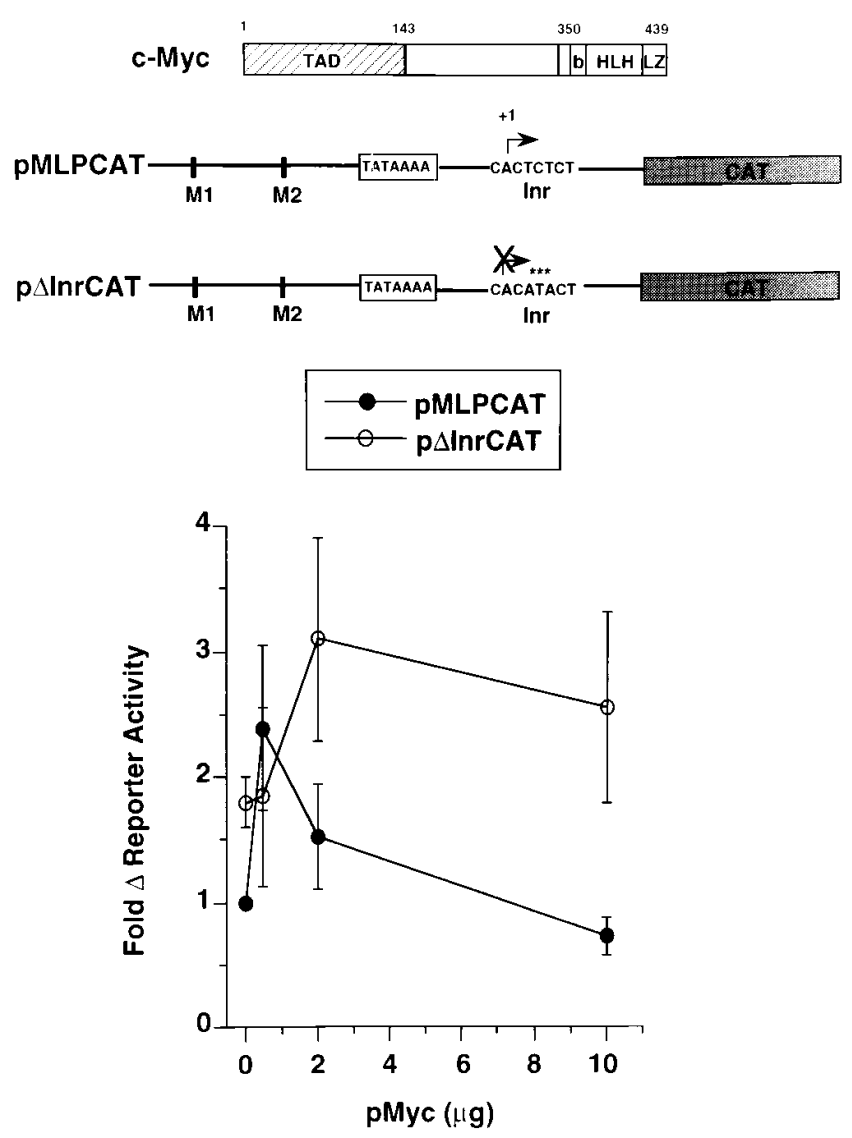

Figure 1. Schematic diagrams of the c-Myc protein (top bar) and reporter constructs are shown at the top. $\mathrm{p} \Delta \mathrm{InrCAT}$ is identical to pMLPCAT with the exception of having a mutated Inr. Asterisks denote nucleotide substitutions in $\mathrm{p} \Delta \mathrm{InrCAT}$. Graph at the bottom of the figure reflects reporter activities with $10 \mu \mathrm{g}$ pMLPCAT (dark circles) or $\mathrm{p} \Delta \mathrm{InrCAT}$ (open circles) as functions of increasing amounts of a Myc expression plasmid, pMyc. Data obtained from triplicate experiments are presented to reflect the average 1.8-fold greater basal activity of $\mathrm{p} \Delta \mathrm{InrCAT}$ as compared to that of pMLPCAT based on 37 separate transfection experiments. Vertical bars indicate standard errors. $T A D$, transactivation domain; $b$, basic region; $H L H$, helix-loophelix motif; $L Z$, leucine zipper motif; $M 1$ and $M 2$, Myc binding sites; Inr, initiator element; $C A T$, chloramphenicol acetyltransferase gene.

fold changes were subjected to statistical analysis to determine their significance.

To delineate the role of the Inr in c-Myc repression, PCRbased mutagenesis was used to render the initiator sequence incapable of binding to TFII-I $(28,47)$. The fragment containing the mutated Inr was subcloned into a CAT expression plasmid to generate a second reporter construct, $\mathrm{p} \Delta$ InrCAT (Fig. 1). The remainder of the promoter region of $\mathrm{p} \Delta \mathrm{InCAT}$ is identical to the adML promoter. p $\Delta$ InrCAT generated basal transcriptional activity 1.8 times greater than that observed with pMLPCAT as determined from an average of 37 separate transfection experiments. A significant rise in activation of $\mathrm{p} \Delta \mathrm{InrCAT}$ with $2.0 \mu \mathrm{g}$ pMyc was observed (Fig. 1). With 10 $\mu \mathrm{g} \mathrm{pMyc}$, the upward trend in transcriptional activity persisted. This trend contrasts with the repression seen with pMLPCAT. Thus, our results indicate that in the presence of the Inr, Myc transactivates pMLPCAT at low input effector DNA and represses transcription of pMLPCAT at high input Myc expres- sion plasmid DNA. In the absence of the Inr, however, high input levels of Myc expression plasmid did not suppress transcription of $\mathrm{p} \Delta \mathrm{InrCAT}$.

Transcriptional repression requires a region involving amino acids 106 to 143 . The c-Myc $\mathrm{NH}_{2}$-terminal transactivation domain is believed to be required for cell transformation. This contradicts the observation that $\mathrm{p} \Delta 106-143$, a mutant Myc plasmid with a deletion in the transactivation domain, fails to transform but retains transactivation ability $(2,5,14)$. c-Myc activity through the Inr may provide some insight into the apparent dissociation of transcriptional activation from transformation observed with this mutant. Cotransfection of $5.0 \mu \mathrm{g} \mathrm{p} \Delta 106-143$ with pMLPCAT caused a dramatic 6.7-fold increase in CAT activity in comparison to only a 1.7 -fold increase seen when cotransfected with p $\Delta$ InrCAT (Fig. 2). Transfection with $\mathrm{pMLV}$, an empty expression plasmid, failed to affect basal transcription (data not shown). These observations suggest that the ability of c-Myc to suppress the adML promoter in an Inr-dependent fashion is abrogated by deletion of amino acids 106 to 143 , thus resulting in a dramatic activation of pMLPCAT by $\mathrm{p} \Delta 106-143$. In contrast, the nontransforming deletion mutant, $\mathrm{p} \Delta \mathrm{HLH}$, was inactive with either pMLPCAT or p InrCAT (Fig. 2). The failure of $\mathrm{p} \Delta \mathrm{HLH}$ to activate either reporter suggests that the HLH region is required for transactivation and transcriptional suppression. The different transcriptional activities of these mutants do not arise
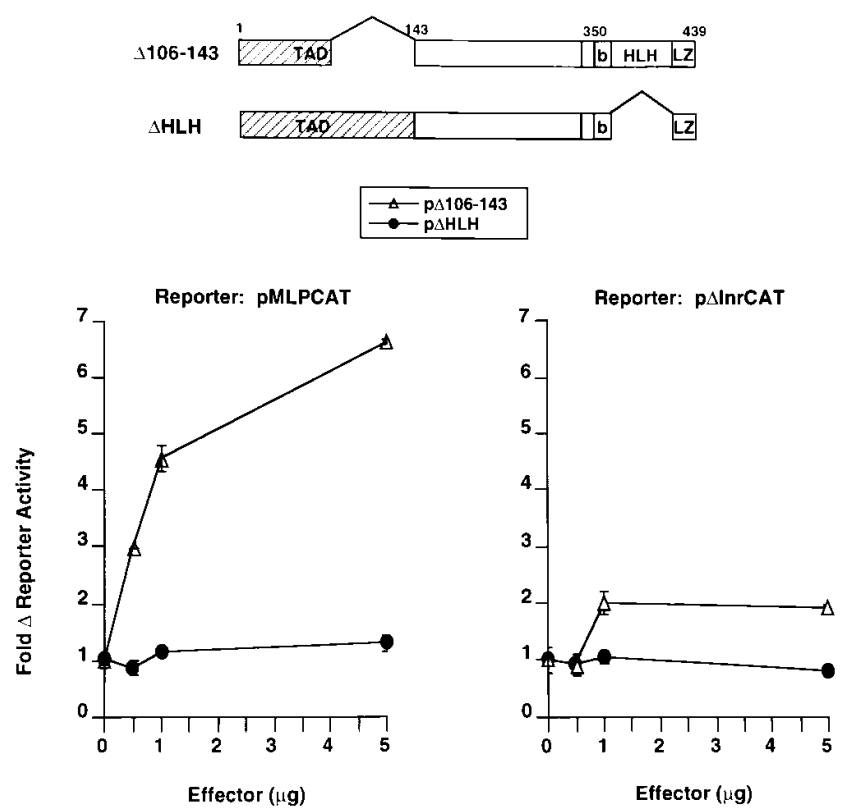

Figure 2. Amino acids 106-143 and the helix-loop-helix $(H L H)$ motif are required for repression of the adML promoter through the initiator element. Schematic diagrams of two Myc deletion mutant proteins, $\Delta 106-143$ and $\Delta \mathrm{HLH}$, are shown at the top of the figure. Graphs at the bottom of the figure show reporter activities with $10 \mu \mathrm{g}$ pMLPCAT or $\mathrm{p} \Delta \mathrm{InrCAT}$ as functions of increasing amounts $\mathrm{p} \Delta 106-$ 143 (open triangles) or $\mathrm{p} \Delta \mathrm{HLH}$ (dark triangles) effector plasmids. Note that $\mathrm{p} \Delta \mathrm{HLH}$ did not alter activities of either pMLPCAT or $\mathrm{p} \Delta$ InrCAT, whereas $\mathrm{p} \Delta 106-143$ activated but did not suppress these reporters. $\mathrm{p} \Delta 106-143$ displayed a marked differential activation of pMLPCAT compared to the moderate activation of $\mathrm{p} \Delta \mathrm{InCAT}$. Transfections were performed in duplicate. Standard error bars are indicated. 
PDMCAT
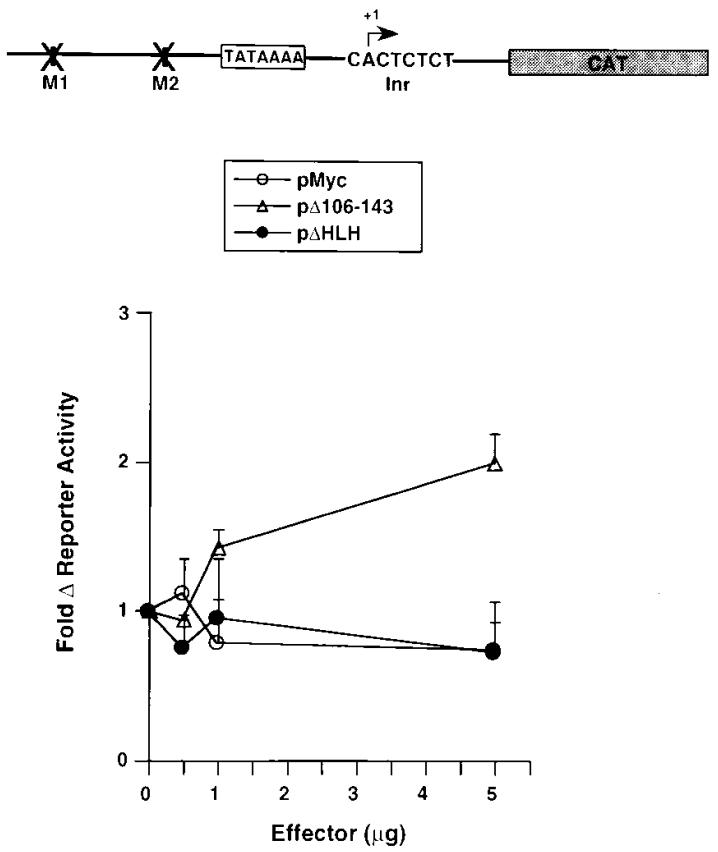

Figure 3. The Myc mutant, $\Delta 106-143$, derepresses the adML promoter through the Inr, independent of the upstream Myc binding sites. At the top of the figure, pDMCAT is illustrated to contain mutations rendering the two Myc binding sites, M1 and M2, defective. The graph depicts reporter activities with $10 \mu \mathrm{g}$ pDMCAT as functions of increasing amounts of pMyc (open circles), p $\Delta 106-143$ (open triangles), or $\mathrm{p} \Delta \mathrm{HLH}$ (closed circles) effector plasmids. Results from triplicate experiments are shown. Vertical bars indicate standard errors.

from differences in expressed protein because the mutant proteins encoded by these plasmids ( $\mathrm{p} \Delta 106-143$ and $\mathrm{p} \Delta \mathrm{HLH}$, the latter also known as $\mathrm{p} \Delta 371-412$ ) have previously been shown to be expressed in transiently and stably transfected cells $(5,14)$.

If loss of region 106-143 simply eliminates transcriptional repression, then transactivation of $\mathrm{p} \Delta \mathrm{InrCAT}$ by $\mathrm{p} \Delta 106-143$ would be expected to occur in a similar pattern as seen with
pMLPCAT. But $\mathrm{p} \Delta 106-143$ exhibited significantly less activation of $\mathrm{p} \Delta \mathrm{InrCAT}$ than of pMLPCAT (Fig. 2). These observations suggest that transactivation is also dependent on the Inr of the adML promoter as previously observed by Du et al. (48). Our observations, however, are unable to rule out the possibility that $\mathrm{p} \Delta 106-143$ behaves in a dominant negative manner by interfering with the inhibitory effect of endogenous Myc through the Inr present in pMLPCAT but not in p $\Delta$ InrCAT. To determine if transactivation by $\mathrm{p} \Delta 106-143$ could occur directly through the Inr independent of the two E-boxes, we mutated both upstream E-boxes of the adML promoter while leaving the Inr intact to generate the reporter construct pDMCAT (Fig. 3). p $\Delta 106-143$ caused a 2.3-fold rise in pDMCAT activity at the highest input effector DNA (Fig. 3). In contrast, neither pMyc nor $\mathrm{p} \Delta \mathrm{HLH}$ affected basal transcription of pDMCAT. Thus, in total these findings suggest that the Inr plays a role in transactivation of MLP and that $\mathrm{p} \Delta 106-143$ is able to transactivate through the Inr in the absence of the two upstream E-boxes.

Deletion of amino acids 106-143 abolished the ability of c-Myc to transform and the ability of c-Myc to repress through the Inr, yet did not eliminate its transactivating property. Unlike $\mathrm{p} \Delta 106-143$ which fails to transform at all $(5,6)$, other $\mathrm{NH}_{2}-$ terminal deletion mutants, $\mathrm{p} \Delta 41-53, \mathrm{p} \Delta 55-92$, and $\mathrm{p} \Delta 93-104$, retained the ability to transform but with diminished efficiency as compared to wild-type Myc in the rat embryo fibroblast cotransformation assay (5). We questioned if derepression through the Inr was unique to deletion of amino acids 106-143 and studied the transcriptional properties of these other deletion mutants. Derepression through the Inr, reflected by the greater activation of pMLPCAT as compared to $\mathrm{p} \triangle \mathrm{InrCAT}$, was exhibited by only two deletion mutants. Derepression through the Inr was greatest with $\mathrm{p} \Delta 106-143$. A mutant with deletion of amino acids 93 to 103, a region contiguous to 106143 , also demonstrated derepression through the Inr but to a lesser degree (Fig. 4). The other two mutants, $\mathrm{p} \Delta 41-53$ and $\mathrm{p} \Delta 55-92$, retained the ability to repress through the Inr because their activation of $\mathrm{p} \Delta \mathrm{InrCAT}$ was greater than that of pMLPCAT. The findings suggest that a region involving amino acids $106-143$ of the transactivation domain is necessary

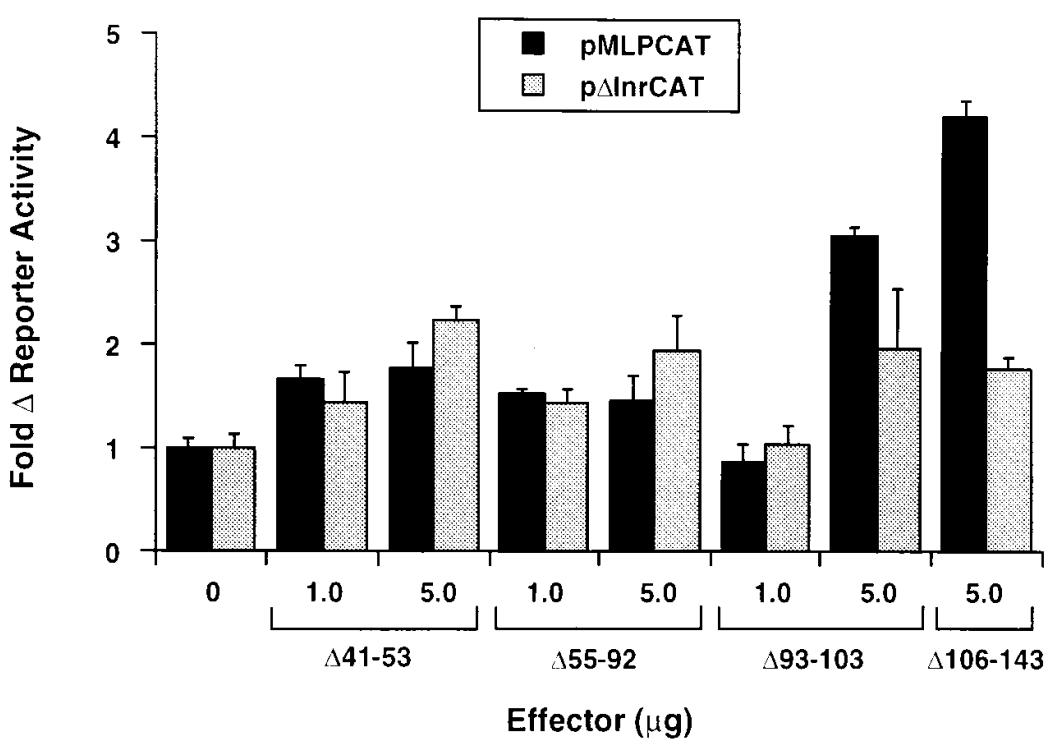

Figure 4. Amino acids 106-143 are necessary for repression through the Inr. Plasmids encoding $\mathrm{NH}_{2}$-terminal deletion mutants, which are indicated at the bottom of the graph by $\Delta$ followed by the deleted codon numbers, were cotransfected in the amounts indicated with either pMLPCAT (black bars) or $\mathrm{p} \Delta \mathrm{InrCAT}$ (shaded bars). Reporter activities are indicated by the vertical axis. Repression through the Inr is indicated by a higher relative activity of $\mathrm{p} \Delta \mathrm{InrCAT}$ compared to pMLPCAT. The converse pattern represents de-repression. Data shown is the average of triplicate experiments. Bars indicate standard errors. 
for c-Myc-mediated transcriptional repression, since loss of this region abrogates the ability of c-Myc to suppress transcription from pMLPCAT as compared to the other deletion mutants. The derepression seen by $\mathrm{p} \Delta 93-103$ suggests that deletion of amino acids 93-103 may alter the conformation of adjacent amino acids 106-143 resulting in the loss of the ability of c-Myc to repress.

Increased transcriptional suppression by a lymphomaderived mutant Myc. Since amino acids 106 to 143 appear to be required for both the transformation and repressive activities of c-Myc, repression could be coupled to transformation. To determine whether a link could be more clearly established between transcriptional and transformation activities, we examined the activity of a lymphoma-derived mutant that contains a mutation within 106 to 143 . At least $65 \%$ of Burkitt lymphomas exhibit missense mutations of c-myc clustered within its transactivation domain $(49,50)$. While sporadic missense mutations that have no functional consequences may result from hypermutability of the translocated c-myc allele, recurrent missense mutations may indicate a growth selection advantage for cells containing such mutations. Mutations clustered around codon 58 have been shown to resist suppression of Myc-mediated transactivation by the retinoblastomarelated protein, p107 $(51,52)$. 4 of 45 lymphoma-derived c-myc alleles display a missense mutation at codon 115 resulting in substitution of a phenylalanine for leucine $(40,50,52)$. Mutation of 115-Phe, however, is still susceptible to p107 suppression of Myc transactivation (Clark, H.M., T. Yano, C.V. Dang,
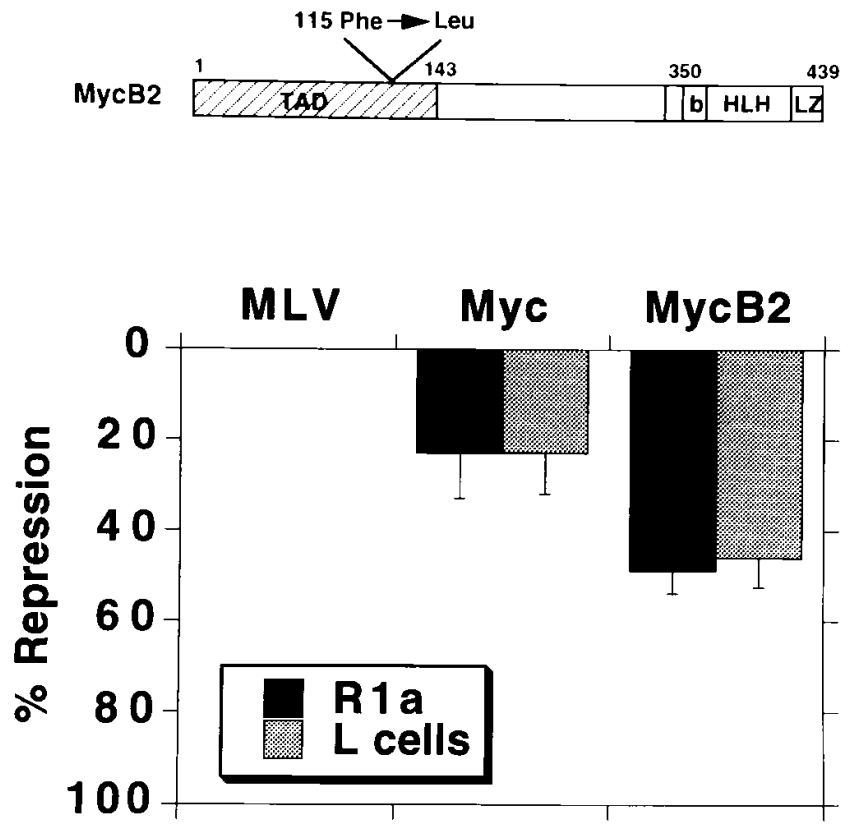

Figure 5. The lymphoma-derived mutant, MycB2, is a stronger repressor of the adML promoter than wild-type Myc. The mutant Myc protein is depicted at the top of the figure. The graph represents repression of pMLPLuc $(10 \mu \mathrm{g})$ reporter activity after cotransfection with either $5 \mu \mathrm{g}$ pMyc or $5 \mu \mathrm{g} \mathrm{pMycB} 2$ (and in the presence of $0.5 \mu \mathrm{g}$ USF for experiments in mouse L cells) as compared to basal activity generated by cotransfection of $5 \mu \mathrm{g}$ pMLV, an empty expression plasmid. Experiments with Rat 1a $(R 1 a)$ cells were performed by lipofection in the absence of the USF expression vector. Quadruplicate experiments were performed. Standard error bars are shown. and M. Raffeld, unpublished observation). We sought to delineate the transcriptional properties of the lymphoma derived mutant, B2, because it contains a substitution at 115-Phe which lies within the region required for transcriptional suppression.

We first compared the activation of $\mathrm{p} \Delta \mathrm{InrLuc}$ by Myc and MycB2 and found that both transactivated $\mathrm{p} \Delta \mathrm{InrLuc}$ similarly (data not shown). Then to determine if their abilities to repress through the Inr were likewise similar, transfections using pMLPLuc were performed. Repression of pMLPLuc by Myc versus MycB2 was studied in the presence of a USF expression plasmid in mouse L cells. The addition of USF raises the basal activity of pMLPLuc twofold and thereby increases the sensitivity to changes in transcriptional repression. USF has been shown to transactivate through both the E-boxes and Inr element of the adML promoter (48). Li et al. have shown that USF raises the activity of the adML promoter, which accentuates the inhibitory effects of overexpressed c-Myc (37). At high effector plasmid input, mutant pMycB2 more strongly suppressed pMLPLuc as compared to wild-type pMyc in mouse L cells (Fig. 5). pMycB2 also suppressed pMLPLuc more potently than wild-type pMyc in Rat 1 a cells that were used in the following experiments to assess the transforming properties of c-Myc (Fig. 5).

We tested whether $\mathrm{pMycB} 2$ is more potent than $\mathrm{pMyc}$ in inducing the neoplastic phenotype by examining their abilities (a) to alter growth properties of Rat 1a cells, $(b)$ to induce anchorage-independent growth of Rat 1a cells (44), and (c) to transform primary rat embryo cells in cooperation with activated EJ-ras (2).

We generated pooled Rat 1a cell lines stably transfected with $\mathrm{pMyc}, \mathrm{pMycB} 2$, or the empty expression vector, pMLV. The Rat 1a cell line was chosen because this immortalized cell line can be transformed by wild-type c-Myc alone resulting in anchorage-independent growth (44). The expression of c-Myc proteins in each of these pools was determined by immunoblot analysis using an antibody specific for human c-Myc (Fig. 6a). The cells were plated in media with $10 \%$ FCS, and $6 \mathrm{~d}$ after plating the $\mathrm{pMycB} 2$ cells began to spontaneously form clusters of rounded cells that could grow in a cell-contact-independent manner, whereas the vast majority of pMLV and pMyc cells remained adherent to the plate (Fig. $6 \mathrm{~b}$ ). The nonadherent pMycB 2 cells were $>95 \%$ viable as determined by trypan blue exclusion (data not shown). Saturation densities of each of the three cell lines (Fig. 7) were obtained for $10 \mathrm{~d}$ and showed that the pMycB2 cells continued to proliferate beyond the values achieved by pMLV and pMyc cells, indicating that MycB2 cells are less susceptible to contact inhibition of growth as is morphologically evident in Fig. $6 b$.

As another indicator of cell transformation, the pooled cell lines were assayed for anchorage-independent growth in soft agarose as described previously (44). The pMyc stably transformed cell line gave rise to colonies of intermediate size, but only few large colonies. In contrast, the pMycB2-transformed cell line formed intermediate colonies $(100-200 \mu \mathrm{m})$, and in addition, large colonies $(>200 \mu \mathrm{m})$ (Fig. $6 \mathrm{~b}$ ). pMLV-transfected cells did not yield colonies $>100 \mu \mathrm{m}$ in size. The observation that large colonies were formed only by the pMycB2stable cell line and the higher cloning efficiency suggest that the amino acid substitution at amino acid 115, which enables c-Myc to have greater transcriptional repressive activity, also imposes a more transformed phenotype. The larger colonies seen with $\mathrm{pMycB} 2$ can be attributed to enhanced anchorage- 


\section{b MLV}
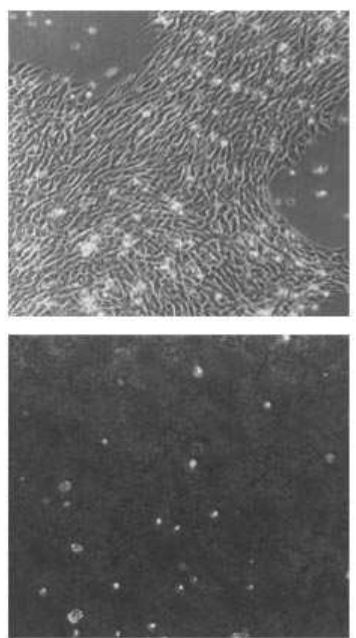

Colony Size \begin{tabular}{l}
$\mathbf{1 0 0 - 2 0 0 \mu \mathrm { m }}$ \\
\hline $\mathbf{2 0 0 \mu \mathrm { m }}$
\end{tabular}
Myc
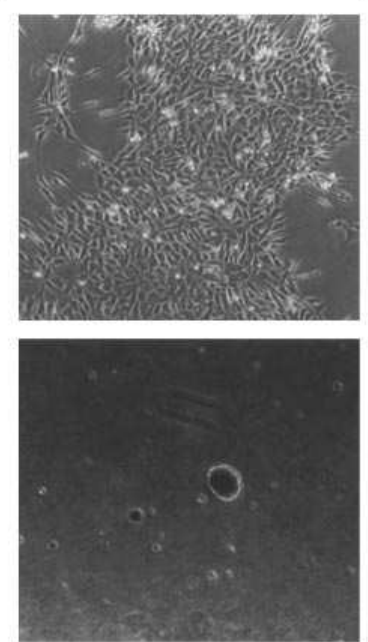

Colony Number
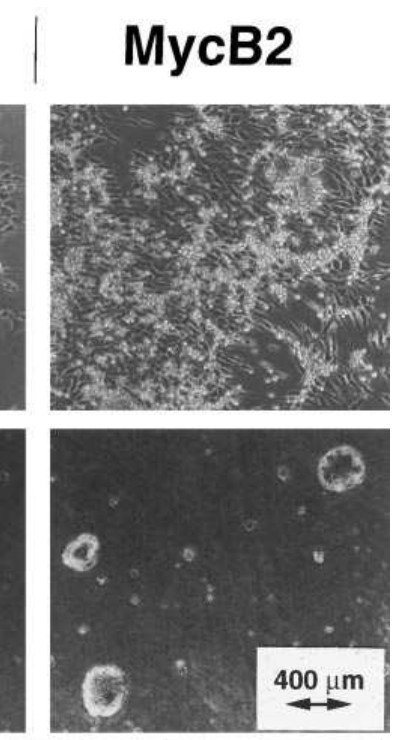

$400 \mu \mathrm{m}$
2

.

Figure 6. The lymphoma-derived MycB2 mutant transforms Rat 1a fibroblasts more effectively than wild-type c-Myc. (a) Western immunoblot of extracts from Rat 1a fibroblasts transfected with either pMyc, pMycB2, or pMLV (an empty expression plasmid). Myc polypeptides in whole cell lysates were resolved on a denaturing $10 \%$ polyacrylamide gel, electroblotted onto a nitrocellulose membrane, and detected with an $\mathrm{mAb}$ specific for human c-Myc (9E10). (b) Top row: MycB2 stably transfected cells lose contact inhibition as compared to control MLV and wild-type Myc. $1.8 \times 10^{5}$ cells were plated in DME with $10 \%$ FCS and photographed on day 7. Bottom row: Soft agarose assay for anchorageindependent growth. Rat 1a cells transfected with pMLV, pMyc, or pMycB2 were plated in $0.7 \%$ agarose as described in Methods. Colonies from duplicate dishes were counted and measured on day 16. Colony size and number per $100-\mathrm{mm}$ plate (mean of two experiments with duplicates in each experiment) are indicated at the bottom of the figure.

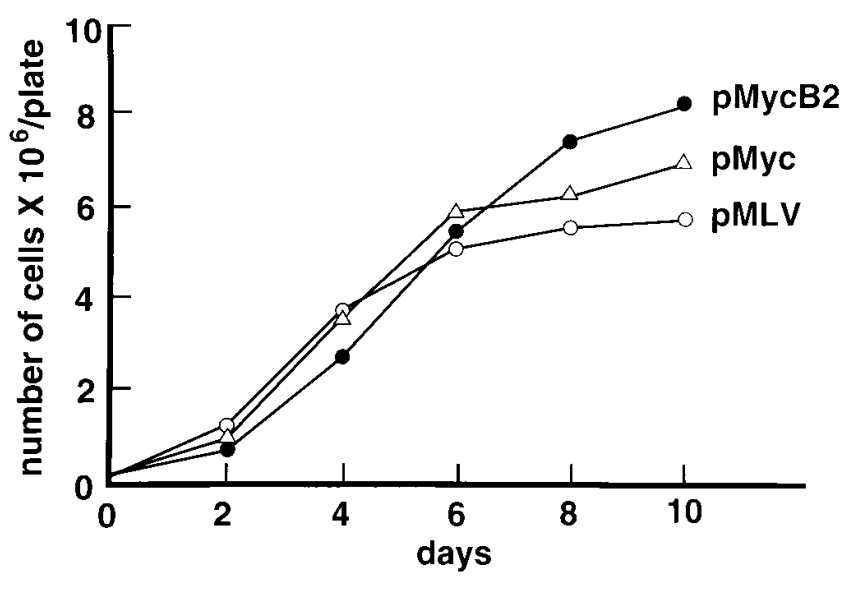

Figure 7. MycB2 stably transfected cells exhibit higher growth saturation density than control MLV or wild-type c-Myc transformed Rat 1a cells. $1.8 \times 10^{5}$ cells/100-mm dish were plated for each condition. Cells on duplicate plates were trypsinized and counted on the days indicated for up to $10 \mathrm{~d}$. Averaged cell numbers per dish are displayed as a function of time in days. independent cell proliferation as opposed to diminished apoptosis, since the extent of apoptotic cell death with serum withdrawal is similar between pMyc and pMycB2 cells (data not shown). These observations suggest that MycB2 is more potent than wild-type c-Myc in promoting anchorage-independent growth of Rat 1a cells.

Through the use of an independent cell transformation assay, we observed that the lymphoma derived MycB2 is more potent than wild-type c-Myc in transforming primary rat embryo cells in cooperation with the activated EJ-ras gene (Table I). The conditions for this assay are as described in our previous publications $(3,53)$. EJ-ras or MycB2 alone did not transform rat embryo cells. Under conditions defined in Table I, lipofected primary rat embryo cells displayed an approximate twofold increase in transformed foci with MycB2 as compared to wild-type c-Myc in triplicate experiments. In these experiments, the total amounts of input plasmid DNA were made constant by adding the empty MLV-LTR-containing plasmid, pMLV. These observations further underscore the higher potency of $\mathrm{MycB} 2$ as compared to wild-type c-Myc. When considered with the finding that $\mathrm{p} \Delta 106-143$ lacks both transforming and transcriptional suppression activities, the observations with $\mathrm{pMycB} 2$ strongly support a link between the transformation and transcriptional suppression by c-Myc. 
Table I. Transformation of Primary Rat Embryo Fibroblasts by Wild-Type c-Myc Versus the Lymphoma-derived MycB2 in Cooperation with EJ-Ras

\begin{tabular}{|c|c|c|c|c|c|}
\hline Plasmid & $\begin{array}{l}\mu \mathrm{g} \text { DNA per } \\
100 \text {-mm dish }\end{array}$ & $\begin{array}{l}\text { Number of foci } \\
\text { Expt. No. } 1\end{array}$ & $\begin{array}{l}\text { Number of foci } \\
\text { Expt. No. } 2\end{array}$ & $\begin{array}{l}\text { Number of foci } \\
\text { Expt. No. } 3\end{array}$ & $\begin{array}{l}\text { Average number } \\
\text { of foci } \pm \text { SD }\end{array}$ \\
\hline pMLVMyc & 10 & 30 & 23 & 24 & $26 \pm 4$ \\
\hline pEJras & 5 & & & & \\
\hline pMLV & 10 & & & & \\
\hline pMLVMycB2 & 10 & 52 & 40 & 46 & $46 \pm 6$ \\
\hline pEJras & 5 & & & & \\
\hline pMLV & 10 & & & & \\
\hline pEJras & 5 & 0 & 0 & 0 & 0 \\
\hline pMLV & 20 & & & & \\
\hline pMLVMycB2 & 10 & 0 & 0 & 0 & 0 \\
\hline pMLV & 15 & & & & \\
\hline
\end{tabular}

Expt., experiment.

\section{Discussion}

This study stemmed from our earlier work to identify factors influencing the transactivation properties of c-Myc. In our previous study (31) we noted that at low cell confluency, high input c-Myc expression plasmid resulted in transcriptional suppression of the adML promoter as was previously observed by van Antwerp et al., who used our reporter construct (29). We now demonstrate that $\mathrm{c}-\mathrm{Myc}$ represses transcription through the initiator element of the adenoviral major late promoter. Titration of c-Myc resulted in transactivation of the adML promoter and subsequent suppression at high levels of Myc. We also show that an $\mathrm{NH}_{2}$-terminal region of c-Myc spanning amino acids 106-143 is required for transcriptional suppression but not transactivation, whereas the helix-loop-helix region is necessary for both transactivation and transcriptional suppression. A lymphoma-derived c-Myc mutant (B2) containing an amino acid substitution within the $\mathrm{NH}_{2}$-terminal region required for transcriptional suppression and transformation is more effective than wild-type c-Myc in transforming Rat 1a fibroblasts and in suppressing the adML promoter. Thus, in addition to defining an $\mathrm{NH}_{2}$-terminal region of c-Myc required for transcriptional suppression of the initiator element, we demonstrate a link between c-Myc-mediated transformation and transcriptional suppression through both loss-of-function and gain-of-function c-myc mutations.

$c$-Myc-mediated transcriptional repression occurs through the Inr and requires an $\mathrm{NH}_{2}$-terminal region and the $\mathrm{HLH}$ dimerization domain. The study of c-Myc-mediated transactivation is complicated by the variable degrees of transactivation that occur through the E-box observed by different investigators $(11-14,29,30)$. Part of this variability could arise from the use of different cell lines in transient transfections. We have recently shown that changes in cell density at the time of transfection also contribute to variation in c-Myc-mediated transactivation because endogenous levels of c-Myc vary inversely with cell density (31). However, differences in cell type and cell density fail to sufficiently explain the reports of c-Mycmediated transcriptional repression $(11,29,30)$.

The observation that Myc could transcriptionally repress the adML promoter at low cell confluency and the reported in- teraction of c-Myc with TFII-I led us to examine its activity through the adML promoter by mutating the initiator element. Constructs encoding Myc repress basal transcription at high inputs in the presence of an intact Inr and intact E-boxes. When the Inr is mutated, however, transcriptional repression is abolished. Thus, we have observed that transcriptional repression by c-Myc can occur through the Inr element in vivo. This observation substantiates the findings that c-Myc could inhibit TFII-I-dependent in vitro transcription (28). Our results are consistent with the recent observation that c-Myc represses the $\lambda 5$ and terminal deoxynucleotidyl transferase initiators in transfection experiments (39).

The inhibitory effect of c-Myc is most evident when compared to the transactivation properties of the mutant $\Delta 106$ 143. In contrast to wild-type c-Myc, which activates and then suppresses transcription of the adML promoter, $\Delta 106-143$ was unable to suppress transcription at high levels of effector plasmid. In fact, $\Delta 106-143$ stimulated the reporter pMLPCAT nearly sevenfold at a level of effector plasmid where wild-type c-Myc suppressed pMLPCAT. The dramatic activation of pMLPCAT by $\Delta 106-143$ may result from the loss of suppressive activity through the Inr and retention of activation through E-boxes or, alternatively, an activation of both the Inr and E-boxes by the mutant $\Delta 106-143$. In contrast to wild-type c-Myc, which did not affect a reporter containing the Inr but lacking two upstream E-boxes (pDMCAT), $\Delta 106-143$ was able to stimulate pDMCAT. This observation suggests that $\Delta 106-143$ interferes with the inhibition of the Inr by endogenous c-Myc through a dominant negative mechanism, although it is possible that it is able to activate transcription through the Inr.

Deletions of similar regions of c-Myc overlapping amino acids 106-143 have been previously shown to result in the loss of transcriptional repression. Deletion of amino acids 41 to 178 was shown to abrogate the ability of c-Myc to repress the mouse metallothionein promoter (30), although the cis element in this promoter responsible for Myc-mediated repression remains as yet unidentified. Furthermore, $\mathrm{Li}$ et al. have also shown that a smaller deletion with removal of amino acids 122-140 resulted in the loss of an ability to suppress the adML promoter (37). In contrast to our study of $\Delta 106-143$, however, deletion of amino acids 122 to 140 did not dramatically aug- 
ment the ability of this mutant to transactivate the adML promoter as compared to wild-type Myc.

The HLH region is required for repression at the Inr by Myc, since the deletion mutant $\mathrm{p} \Delta \mathrm{HLH}$ was unable to either activate or suppress pMLPCAT. $\mathrm{p} \Delta \mathrm{HLH}$ also did not affect either pDMCAT or $\mathrm{p} \Delta \mathrm{InrCAT}$. These results indicate that the $\mathrm{HLH}$ region is required in vivo for suppression at the Inr suggesting that an interaction between Myc and some other protein is required. The role of Max in Myc-mediated suppression at the Inr is as yet unclear (37). Several other proteins, such as YY1 and TFII-I, have been shown to interact with the c-Myc $\mathrm{COOH}$-terminal or HLH region. The transcription factor, YY1, a zinc finger protein, associates with the carboxy terminus of Myc in the yeast two hybrid system (27). Myc inhibits both the repressor and activator functions of YY1 whose DNA binding site resembles an initiator element. The YY1 binding site, however, does not follow the adML Inr consensus sequence, and YY1 does not bind to the adML promoter (54). An alternative partner protein could be the ubiquitous transcription factor, TFII-I, which binds to the initiator element as well as to c-Myc in gel-shift assay experiments (28). Deletion of the c-Myc HLH region in those studies results in loss of binding to TFII-I as well as loss of transcriptional repression in vitro. The c-Myc HLH dimerization domain may facilitate binding to TFII-I which possesses a repeat of helices. It is conceivable that interaction of c-Myc with TFII-I at the Inr may position c-Myc in such a way to allow contact of its activation domain with TATA-binding protein at the TATA box to form an inhibitory complex $(24,25)$.

c-Myc transcriptional properties and neoplastic transformation. Before the recent findings that $\mathrm{c}-\mathrm{Myc}$ is capable of transcriptional repression, the transforming activity of c-Myc was thought to be only linked to its ability to activate transcription. Several observations, when taken together, suggest that the transformation and transactivation activities of c-Myc may be dissociated. When the transactivation domain of c-Myc was first localized by using hybrid GAL4 proteins, it was recognized that the mutant $\Delta 106-143$ could potently activate transcription when tethered to GAL4 (2). $\Delta 106-143$ is also capable of activating a reporter construct containing E-boxes from the ornithine decarboxylase gene (14). This mutant, however, is defective in transforming either Rat 1a or primary rat embryo cells (5). In fact, $\Delta 106-143$ or deletion mutants lacking this region have been shown to act in a dominant negative fashion over the wild-type c-myc allele in transformation assays (53, 55). Furthermore, although the chimeric VP16-Myc protein containing the activation domain of VP16 fused to the Myc oligomerization domain is capable of dimerizing with Max (16) and activating transcription of reporter constructs bearing E-boxes, this VP16-Myc chimera was unable to transform rat embryo cells in cooperation with an activated RAS (Dang, C.V., and J. Barrett, unpublished observation). These findings together suggest that the ability of c-Myc to transactivate artificial reporter constructs containing E-boxes can be dissociated from the transforming activity of c-Myc.

If transactivation by c-Myc can be dissociated from c-Myc transforming activity, then how are c-Myc transcriptional properties related to its neoplastic transformation activities? It is probable that artificial reporter constructs bearing E-boxes do not reflect the complexity of transcriptional regulation through authentic promoters in the genome that are regulated by c-Myc. The testing of this hypothesis, however, will require identification and thorough characterization of authentic c-Myc target promoters. An alternative hypothesis could be that transactivation is not the only mechanism by which c-Myc transforms cells.

The biological significance of $c$-Myc-mediated transcriptional suppression. The potential biological significance of transcriptional suppression by c-Myc has been elegantly discussed by Li et al. (37), however several points deserve emphasis. E-box-driven transcription by c-Myc may indeed be biologically important because c-Myc appears to upregulate the expression of at least two growth-related genes, ornithine decarboxylase (14) and prothymosin- $\alpha$ (56). Transactivation of these genes by c-Myc appears to be E-box driven, thus supporting the hypothesis that transactivation of genes involved in cell proliferation is a mechanism by which c-Myc transforms cells.

The link between c-Myc-mediated transcriptional suppression and transformation, however, argues that c-Myc-mediated transactivation of growth-related genes may not be sufficient for cell transformation. We have shown that loss of an $\mathrm{NH}_{2}$-terminal region critical for cell transformation is accompanied by a loss of transcriptional repression, but not transactivation. Our observation that the mutation in MycB2 confers increased transcriptional suppression and transformation activity supports a significant role for transcriptional repression in c-Myc-mediated cell transformation. Since c-Myc induces cell proliferation and transformation, candidate genes that are suppressed by c-Myc are expected to be involved in cell differentiation or cell growth arrest. In fact, several putative target genes, such as albumin, C/EBP- $\alpha$, metallothionein-1, LFA-1, and class I MHC genes, are repressed by c-Myc, and all of these genes contain initiator elements that match the adML Inr consensus sequence. $\mathrm{Li}$ et al. have shown that c-Myc can repress both the albumin and $\mathrm{C} / \mathrm{EBP}-\alpha$ promoters through their initiator elements in reporter constructs (37). Because the genes that are repressed by c-Myc are highly expressed in differentiated cells, it seems plausible that cell transformation by c-Myc involves the down-regulation of differentiation genes. Thus, c-Myc-mediated transformation is a complex process, likely requiring E-box-driven transactivation of growthrelated genes and Inr-driven transcriptional repression of differentiation-related genes.

\section{Acknowledgments}

This work was supported in part by National Institutes of Health grants CA-57341 and CA-51497 (C.V. Dang), an American Gastroenterological Association Advanced Research Training Award (L.A. Lee), and the Rogers-Wilbur Foundation. C.V. Dang is a Scholar of the Leukemia Society of America. L.A. Lee is an A. Guerrieri Clinician-Scientist.

\section{References}

1. Kato, G.J., and C.V. Dang. 1992. Function of the c-Myc oncoprotein. FASEB J. 6:3065-3072.

2. Kato, G.J., J. Barrett, M. Villa-Garcia, and C.V. Dang. 1990. An amino terminal c-myc domain required for neoplastic transformation activates transcription. Mol. Cell. Biol. 10:5914-5920.

3. Dang, C.V., J. Barrett, M. Villa-Garcia, L.M.S. Resar, G.J. Kato, and E.R. Fearon. 1991. Intracellular leucine zipper interactions suggest c-Myc hetero-oligomerization. Mol. Cell. Biol. 11:954-962.

4. Prendergast, G.C., D. Lawe, and E.B. Ziff. 1991. Association of Myn, the murine homolog of Max with c-myc stimulates methylation-sensitive DNAbinding and ras cotransformation. Cell. 65:395-407. 
5. Stone, J., T. de Lange, G. Ramsay, E. Jokobovits, J.M. Bishop, H.E. Varmus, and W.M.F. Lee. 1987. Definition of regions in human c-myc that are involved in transformation and nuclear localization. Mol. Cell. Biol. 7:1697-1709.

6. Sarid, J., T.D. Halazonetis, W. Murphy, and P. Leder. 1987. Evolutionary conserved regions of the human c-Myc protein can be uncoupled from transforming activity. Proc. Natl. Acad. Sci. USA. 84:170-173.

7. Amati, B., M.W. Brooks, N. Levy, T.D. Littlewood, G.I. Evan, and H. Land. 1993. Oncogenic activity of the c-Myc protein requires dimerization with Max. Cell. 72:233-245.

8. Blackwell, T.K., J. Huang, A. Ma, L. Kretzner, F.W. Alt, R.N. Eisenman, and H. Weintraub. 1993. Binding of Myc proteins to canonical and noncanonical DNA sequences. Mol. Cell. Biol. 13:5216-5224.

9. Blackwood, E.M., and R.N. Eisenman. 1991. Max: a helix-loop-helix zipper protein that forms a sequence-specific DNA-binding complex with Myc. Science (Wash. DC). 251:1211-1217.

10. Reddy, C.D., P. Dasgupta, P. Saikumar, H. Dudek, F.J. Rauscher III, and E.P. Reddy. 1992. Mutational analysis of Max: role of basic, helix-loophelix/leucine zipper domains in DNA binding, dimerization and regulation of Myc-mediated transcriptional activation. Oncogene. 7:2085-2092.

11. Kretzner, L., E.M. Blackwood, and R.N. Eisenman. 1992. Myc and Max proteins possess distinct transcriptional activities. Nature (Lond.). 359:426-429.

12. Gu, W., K. Cechova, V. Tassi, and R. Dalla-Favera. 1993. Opposite regulation of gene transcription and cell proliferation by c-Myc and Max. Proc. Natl. Acad. Sci. USA. 90:2935-2939.

13. Amin, C., A. Wagner, and N. Hay. 1993. Sequence-specific transcriptional activation by Myc and repression by Max. Mol. Cell. Biol. 13:383-390.

14. Bello-Fernandez, C., G. Packham, and J.L. Cleveland. 1993. The ornithine decarboxylase gene is a transcriptional target of c-Myc. Proc. Natl. Acad. Sci. USA. 90:7804-7808.

15. Berberich, S., N. Hyde-DeRuyscher, P. Espenshade, and M. Cole. 1992. Max encodes a sequence-specific DNA-binding protein and is not regulated by serum growth factors. Oncogene. 7:775-779.

16. Kato, G.J., W.M.F. Lee, L. Chen, and C.V. Dang. 1992. Max: functional domains and interaction with c-Myc. Genes Dev. 6:81-92.

17. Ayer, D.E., L. Kretzner, and R.N. Eisenman. 1993. Mad: a heterodimeric partner for Max that antagonizes Myc transcriptional activity. Cell. 72:211-222.

18. Zervos, A., J. Gyuris, and R. Brent. 1993. Mxi1, a protein that specifically interacts with Max to bind Myc-Max recognition sites. Cell. 72:223-232.

19. Lahoz, E.G., L. Xu, N. Schreiber-Agus, and R.A. DePinho. 1994. Suppression of Myc but not E1A transformation activity by Max-associated proteins, Mad and Mxi-1. Proc. Natl. Acad. Sci. USA. 91:5503-5507.

20. Schreiber-Agus, N., R. Torres, J. Horner, A. Lau, M. Jamrich, and R.A. DePinho. 1993. Comparative analysis of the expression and oncogenic activities of Xenopus c-, N-, and L-myc homologs. Mol. Cell. Biol. 13:2456-2468.

21. Blackwood, E.M., B. Lüscher, and R.N. Eisenman. 1992. Myc and Max associate in vivo. Genes Dev. 6:71-80.

22. Wagner, A., M.M. Le Beau, M.O. Diaz, and N. Hay. 1992. Expression, regulation and chromosomal localization of the Max gene. Proc. Natl. Acad. Sci. USA. 89:3111-3115.

23. Ayer, D.E., and R.N. Eisenman. 1993. A switch from Myc:Max to Mad: Max heterocomplexes accompanies monocyte/macrophage differentiation. Genes Dev. 7:2110-2119.

24. Maheswaran, S., H. Lee, and G. Sonenshein. 1994. Intracellular association of the protein product of the c-myc oncogene with the TATA-binding protein. Mol. Cell. Biol. 14:1147-1152.

25. Hateboer, G., H.T.M. Timmers, A.K. Rustgi, M. Billaud, L.J. van't Veer, and R. Bernards. 1993. TATA-binding protein and the retinoblastoma gene product bind to overlapping epitopes on c-Myc and adenovirus E1A protein. Proc. Natl. Acad. Sci. USA. 90:8489-8493.

26. Beijersbergen, R.L., E.M. Hijmans, and R. Bernards. 1994. Interaction of c-Myc with the pRb-related protein p107 results in inhibition of c-Myc-mediated transactivation. EMBO (Eur. Mol. Biol. Organ.) J. 13:4080-4086.

27. Shrivastava, A., S. Saleque, G.V. Kalpana, S. Artandi, S.P. Goff, and K. Calame. 1993. Inhibition of transcriptional regulator Yin-Yang-1 by association with c-Myc. Science (Wash. DC). 262:1889-1892.

28. Roy, A.L., C. Carruthers, T. Gutjahr, and R.G. Roeder. 1993. Direct role for Myc in transcription initiation mediated by interactions with TFII-I. Nature (Lond.). 365:359-361.

29. Van Antwerp, M.E., D.G. Chen, C. Chang, and E.V. Prochownik. 1992. A point mutation in the MyoD basic domain imparts c-Myc-like properties. Proc. Natl. Acad. Sci. USA. 89:9010-9014.

30. Kaddurah-Daouk, R., J.M. Greene, A.S. Baldwin, Jr., and R.E. Kingston. 1987. Activation and repression of mammalian gene expression by the c-myc protein. Genes Dev. 1:347-357.

31. Lee, L.A., L.M.S. Resar, and C.V. Dang. 1994. Cell density and paradoxical transcriptional properties of c-Myc and Max in cultured mouse fibro- blasts. J. Clin. Invest. 95:900-904.

32. Roy, B., J. Beamon, E. Balint, and D. Reisman. 1994. Transactivation of the human p53 tumor suppressor gene by c-Myc/Max contributes to elevated mutant p53 expression in some tumors. Mol. Cell. Biol. 14:7805-7815.

33. Yang, B.-S., T.J. Geddes, R.J. Pogulis, B. de Crombrugghe, and S.O Freytag. 1991. Transcriptional suppression of cellular gene expression by c-Myc. Mol. Cell. Biol. 11:2291-2295.

34. Jansen-Durr, P., A. Meichle, P. Steiner, M. Pagano, D. Finke, J. Botz, J. Wessbecher, G. Draetta, and M. Eilers. 1993. Differential modulation of cyclin gene expression by MYC. Proc. Natl. Acad. Sci. USA. 90:3685-3689.

35. Corden, J., B. Wasylyk, A. Buchwalder, P. Sassone-Corsi, C. Kedinger, and P. Chambon. 1980. Promoter sequences of eukaryotic protein-encoding genes. Science (Wash. DC). 209:1405-1414.

36. Smale, S.T., and D. Baltimore. 1989. The "initiator" as a transcription control element. Cell. 57:103-113.

37. Li, L.-H., C. Nerlov, G. Prendergast, D. MacGregor, and E.B. Ziff. 1994. c-Myc represses transcription in vivo by a novel mechanism dependent on the initiator element and Myc box II. EMBO (Eur. Mol. Biol. Organ.) J. 13: 4070-4079.

38. Philipp, A., A. Schneider, I. Vasrik, K. Finke, Y. Xiong, D. Beach, K. Alitalo, and M. Eilers. 1994. Repression of cyclin D1: a novel function of $m y c$. Mol. Cell. Biol. 14:4032-4043.

39. Mai, S., and I.-L. Mårtensson. 1995. The c-myc protein represses $\lambda 5$ and TdT initiators. Nucleic Acids Res. 23:1-9.

40. Clark, H.M., T. Yano, T. Otsuki, E.S. Jaffe, D. Shibata, and M. Raffeld. 1994. Mutations in the coding region of c-MYC in AIDS-associated and other aggressive lymphomas. Cancer Res. 54:3383-3386.

41. Ausabel, F.M., R. Brent, R.E. Kingston, D.D. Moore, J.G. Seidman, J.A. Smith, and K. Struhl. 1987. Introduction of DNA into mammalian cells. In Current Protocols in Molecular Biology. Vol. 1. John Wiley \& Sons Inc., New York. 9.0.1-9.5.5.

42. Seed, B., and J.Y. Sheen. 1988. A simple phase-extraction assay for chloramphenicol acetyltransferase activity. Gene. 67:271-277.

43. Bradford, M.M. 1976. A rapid and sensitive method for the quantitation of microgram quantities of protein utilizing the principle of protein dye binding. Anal. Biochem. 72:248-254.

44. Hoang, A.T., K.J. Cohen, J.F. Barrett, D.A. Bergstrom, and C.V. Dang. 1994. Participation of cyclin A in Myc-induced apoptosis. Proc. Natl. Acad. Sci. USA. 91:6875-6879.

45. Laemmli, U.K. 1970. Cleavage of structural proteins during the assembly of the head of bacteriophage T4. Nature (Lond.). 227:680-685.

46. Evan, G.I., G.K. Lewis, G. Ransay, and J.M. Bishop. 1985. Isolation of monoclonal antibodies specific for human c-myc proto-oncogene product. Mol. Cell. Biol. 5:3610-3616.

47. Roy, A.L., M. Meisterernst, P. Pogenec, and R.G. Roeder. 1991. Cooperative interaction of an initiator-binding transcription initiation factor and the helix-loop-helix activator USF. Nature (Lond.). 354:245-248.

48. Du, H., A.L. Roy, and R.G. Roeder. 1993. Human transcription factor USF stimulates transcription through the initiator element of the HIV-1 and the adML promoters. EMBO (Eur. Mol. Biol. Organ.) J. 12:501-511.

49. Bhatia, K., K. Huppi, G. Spangler, D. Siwarski, R. Iyer, and I. Magrath. 1993. Point mutations in the c-Myc transactivation domain are common in Burkitt's lymphoma and mouse plasmacytoma. Nat. Genet. 5:56-61.

50. Yano, T., C. Sander, H. Clark, M. Dolezal, E. Jaffe, and M. Raffeld. 1993. Clustered mutations in the second exon of the MYC gene in sporadic Burkitt's lymphoma. Oncogene. 8:2741-2748.

51. Gu, W., K. Bhatia, I.T. Magrath, C.V. Dang, and R. Dalla-Favera. 1994. Binding and suppression of the Myc transcriptional activation domain by 107 . Science (Wash. DC). 264:251-254.

52. Hoang, A., B. Lutterbach, B.C. Lewis, T. Yano, T. Chou, J. Barrett, M. Raffeld, S.R. Hann and C.V. Dang. 1995. A link between increased transforming activity of lymphoma-derived MYC mutant alleles, their defective regulation by $\mathrm{p} 107$, and altered phosphorylation of the c-Myc transactivation domain Mol. Cell. Biol. 15:4031-4042.

53. Dang, C.V., M. McGuire, M. Buckmire, and W.M.F. Lee. 1989. Involvement of the 'leucine zipper' region in the oligomerization and transforming activity of human c-myc protein. Nature (Lond.). 337:664-666.

54. Usheva, A., and T. Shenk. 1994. TATA-binding protein-independent initiation: YY1, TFIIB, and RNA polymerase II direct basal transcription on supercoiled template DNA. Cell. 76:1115-1121.

55. Mukherjee, B., S.D. Morgenbesser, and R.A. DePinho. 1992. Myc family oncoproteins function through a common pathway to transform normal cells in culture: cross-interference by Max and trans-acting dominant mutants. Genes Dev. 6:1480-1492.

56. Eilers, M., S. Schirm, and J.M. Bishop. 1991. The MYC protein activates transcription of the alpha-prothymosin gene. EMBO (Eur. Mol. Biol. Organ.) J. 10:133-141. 